\title{
Functional constraints and evolutionary dynamics of the repeats in the rDNA internal transcribed spacer 2 of members of the Anopheles barbirostris group
}

\author{
Claudia Caterina Paredes-Esquivel ${ }^{1,2}$ and Harold Townson ${ }^{1 *}$
}

\begin{abstract}
Background: The Anopheles barbirostris group is widely distributed in Southeast Asia. Although seven species have been formally described, a molecular analysis of the rDNA ITS2 and the mitochondrial cytochrome oxidase I gene suggests that the group includes species that are morphologically very similar or identical.

We have previously shown that species in the Anopheles barbirostris Subgroup have an exceptionally large ITS2 (>1.5 kb), greater than in any other Anopheline group. However, the molecular processes responsible for generating such a large ITS2 have not previously been explored.

Methods: To determine the processes by which this large ITS2 is generated, we examined the sequence and secondary structure of the ITS2 of 51 specimens from five species of the Anopheles barbirostris Subgroup. These include the anthropophilic species An. campestris and three morphospecies of the Barbirostris Complex: An. vanderwulpi, An. barbirostris I and III, together with a previously undescribed member of this group (Clade IV).

Results and conclusions: All the specimens were found to have an ITS2 greater than $1.5 \mathrm{~kb}$ in length. The possibility that the spacer sequences amplified were pseudogenes was examined and discarded. The large size of ITS2 in the species studied is due to the presence of internal repeats of approximately $110 \mathrm{bp}$ in length, confined to the central region of the spacer. Repeats varied markedly between the species examined, with respect to their organization, number and sequence similarity. The nucleotide diversity increased in direct relation to size variation and the presence of non-repeated elements.

A secondary structure analysis showed that the repeats form hairpin structures with a wide range of free energy values. These hairpin structures are known to facilitate the subsequent processing of mature rRNA. An analysis of the repeats from the different species suggests they originate from a common ancestor, with the repeats appearing before speciation of the Barbirostris Group.
\end{abstract}

Keywords: Anopheles barbirostris, ITS2, Secondary structure, Repeat, DNA insertion

\section{Background}

In the ribosomal genome, the first and second internal transcribed spacers (ITS1 and ITS2) are located between the genes coding for $18 \mathrm{~S}, 5.8 \mathrm{~S}$ and $28 \mathrm{~S}$ in the ribosomal DNA cluster. Although spacer sequences are not present in the mature ribosomal RNA molecules, they mediate

\footnotetext{
* Correspondence: htownson@liverpool.ac.uk

'Department of Vector Biology, Liverpool School of Tropical Medicine,

Pembroke Place, L3 5QA Liverpool, United Kingdom

Full list of author information is available at the end of the article
}

the cleavage of the great RNA precursor [1] and form stable secondary structures [2]. There is evidence that the deletion of ITS2 affects the maturation of both the small and large subunit rRNA [3]. Whilst many mutations become fixed in a population through natural selection and genetic drift, the non-coding spacer regions in ITS2 evolve through a pattern of concerted evolution [4]. Typically, ITS2 exhibits a low level of sequence variation within species and a high level of divergence between species. It is this feature that renders the region

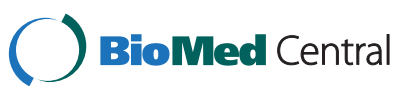

(c) 2014 Paredes-Esquivel and Townson; licensee BioMed Central Ltd. This is an Open Access article distributed under the terms of the Creative Commons Attribution License (http://creativecommons.org/licenses/by/2.0), which permits unrestricted use, distribution, and reproduction in any medium, provided the original work is properly credited. The Creative Commons Public Domain Dedication waiver (http://creativecommons.org/publicdomain/zero/1.0/) applies to the data made available in this article, unless otherwise stated. 
useful to infer phylogenetic relationships in different taxa, including insects [5]. In Anopheles species, ITS2 has proved valuable for distinguishing members of sibling species complexes [6-8] and is the most commonly used marker for the design of species-specific PCR primers for species discrimination [9].

The Anopheles barbirostris group is distributed in the oriental region [10]. Some of its members have been implicated in malaria and filariasis transmission in Southeast Asia [11-13]. Seven species have been formerly described: An. barbirostris, An. campestris, An. donaldi, An. pollicaris, An. franciscoi, An. hodgkini [14] and An. vanderwulpi [15], the latter species being found in sympatry with $A n$. barbirostris sensu stricto in Eastern Java. Anopheles campestris is considered the most anthropophilic of these species. Variations in habitat, resting behaviour and feeding preferences have been reported in the Anopheles barbirostris complex [16-18]. A molecular analysis of ITS2 and the cytochrome oxidase I (COI) gene region confirmed that the morphospecies An. barbirostris comprises at least three morphologically identical species, including An. vanderwulpi. In addition, one species, (Clade IV of Paredes-Esquivel, 2009), shares morphological characters with An. barbirostris and An. campestris.

All species in the Barbirostris Subgroup have a large ITS2 ( $>1.5 \mathrm{~kb})$. The size varies between Clades, namely: Clade I, 1545 bp; Clade II, 1727 bp; Clade III, 1730 bp; Clade IV, 1583 bp and An. campestris, 1519 bp. ITS2 sequences of considerable size have been documented in other Anopheles species. Anopheles crucians species B has an ITS2 of $1012 \mathrm{bp}$ [8]. This length was the result of a complex array of repeats in different combinations. In Anopheles beklemishevi, a member of the Maculipennis Group, ITS2 comprises 638 bp, including two repeats of about 140 bp of similar though not identical sequence [19]. Similarly, An. fluminensis from Bolivia has been reported to have three repeats of 125 bp each within an ITS2 of 596 bp [20]. In Anopheles species of the genus Cellia [21], a series of internal repeats has led to a large ITS1. ITS2 of considerable length have also been seen in other insect species $[8,22,23]$.

In this paper we establish that the outstanding size of ITS2 in the Barbirostris Subgroup is due to the presence of DNA insertions that occur as repeated units located in the central region of the internal transcribed spacer. These vary in copy numbers and organization. To determine whether these mutations affect the stability of the ITS2 and to discard the possibility that they were pseudogenes, i.e. non-functional relatives of genes, we analysed their secondary structure. We have carried out a comprehensive analysis of these repeated elements and discuss their origin in relation to the evolutionary history of the Barbirostris Group members.

\section{Methods}

The species examined were members of the Barbirostris Group studied by Paredes-Esquivel et al. (2009): Anopheles barbirostris clade I collected from Kalimantan (Indonesia) and Mae Hong Son (Thailand); An. vanderwulpi [15] from the island of Sumatra (Indonesia); An. barbirostris clade III, collected from Mae Hong Son, Sa Kaeo, Tak and Kanchanaburi (Thailand); an unknown species clade IV, with mixed characters between $A n$. barbirostris and An. campestris [10] found in Sumatra and Trat and Sa Kaeo (Thailand) and finally An. campestris collected in Sa Kaeo. These species were identified based on the analysis of the COI, ITS2, combined with morphological examination of adult specimens. GenBank accession numbers, sequence sizes and place of collection are summarized in Table 1. Detailed DNA extraction and amplification procedures are described in Paredes-Esquivel et al. (2009).

Sequence alignment was complicated by the presence of internal repeats. BioEdit v 5.0.6. (Hall 1999) was used to align sequences manually and to determine GC content and length. Boundaries of the ITS2 region were identified in comparison to sequences from Anopheles gambiae (GenBank accession number X67157.1) [24]. When the regions containing the repeats were removed, sequences could be aligned with ClustalW [25]. The substitutional rate in the conserved 5,8S and $28 \mathrm{~S}$ regions was analysed in detail to discriminate functional genes from pseudogenes. Repeats were found using the Tandem repeats finder program: http://tandem.bu.edu/trf/ trf.html, although an exhaustive visual examination was also required. Nucleotide diversity $(\pi)$ was calculated with program DNASP5.101 [26] to determine the degree of polymorphism within each type of repeat. This is defined as the average number of pairwise nucleotide substitutions divided by the length of the sequence $(\pi=\Pi / L)$ [27].

Sequences were folded using RNA Folding Form (version 2.3 energies), from the Mfold web server [28], located at: http://mfold.rna.albany.edu/?q=mfold/RNAFolding-Form 2.3. Default parameters $\left(37^{\circ} \mathrm{C}\right.$ with $5 \%$ suboptimal folding) were used to fold sequences. This program provides with several tentative secondary structures for a wide range of free energy values. Only the ITS2 region was included in the analysis.

BLAST searches were carried out to identify sequence similarities. A Bayesian analysis using Mr Bayes 3.2 [29] was carried out to determine phylogenetic relationships among repeats. For this analysis, the Monte Carlo (MCMC) chain length involved 500,000 generations, with trees sampled every 100 generations. The analysis was repeated twice to confirm topologies. Posterior probabilities were employed to test statistical support for clades. Trees were visualized using the FigTree program http://tree.bio.ed.ac.uk/software/figtree/). 
Table 1 List of specimens and size of ITS2 (bp)

\begin{tabular}{|c|c|c|c|c|}
\hline & Specimen & GenBank Acc $\mathbf{N}^{\circ}$ & ITS2 size(bp) & Locality \\
\hline & k2 & EU812759 & 1545 & Kalimantan (Indonesia) \\
\hline & k3 & EU812760 & 1544 & $"$ \\
\hline An. barbirostris & th1.1 & EU812761 & 1544 & Mae Hong Son (Thailand) \\
\hline \multirow[t]{4}{*}{ Clade I } & th 1.3 & EU812764 & 1542 & $"$ \\
\hline & th 1.4 & EU812762 & 1542 & $"$ \\
\hline & th 1.7 & EU812763 & 1542 & $"$ \\
\hline & 112 & EU812766 & 1727 & Sumatra (Indonesia) \\
\hline \multirow[t]{11}{*}{ An. vanderwulpi } & 113 & EU812765 & 1727 & $"$ \\
\hline & 115 & EU812767 & 1727 & $"$ \\
\hline & 133 & EU812768 & 1727 & $"$ \\
\hline & th39.3 & EU812781 & 1732 & Mae Hong Son (Thailand) \\
\hline & th1.6 & EU812776 & 1732 & $"$ \\
\hline & th1.2 & EU812775 & 1733 & $"$ \\
\hline & th 1.8 & EU812782 & 1733 & $"$ \\
\hline & th1.9 & EU812780 & 1732 & $"$ \\
\hline & th1.10 & EU812779 & 1732 & $"$ \\
\hline & bsk33 & EU812769 & 1733 & Sa Kaeo (Thailand) \\
\hline & bsk5 & EU812790 & 1733 & $"$ \\
\hline An. barbirostris & S17.1 & EU812770 & 1733 & $"$ \\
\hline \multirow[t]{21}{*}{ Clade III } & S11.2 & EU812778 & 1733 & " \\
\hline & $\mathrm{S} 24.3$ & EU812789 & 1731 & $"$ \\
\hline & ta10 & EU812785 & 1733 & Tak (Thailand) \\
\hline & ta19 & EU812774 & 1733 & $"$ \\
\hline & ta21 & EU812771 & 1734 & $"$ \\
\hline & ta22 & EU812772 & 1733 & " \\
\hline & ta23 & EU812784 & 1733 & $"$ \\
\hline & ta24 & EU812783 & 1732 & “ \\
\hline & kh3 & EU812787 & 1732 & Kanchanaburi (Thailand) \\
\hline & kh4 & EU812786 & 1733 & $"$ \\
\hline & kh7 & EU812773 & 1732 & " \\
\hline & kh9 & EU812777 & 1732 & $"$ \\
\hline & kh10 & EU812788 & 1732 & " \\
\hline & 114 & EU812791 & 1581 & Sumatra (Indonesia) \\
\hline & btr7 & EU812795 & 1584 & Trat (Thailand) \\
\hline & btr8 & EU812798 & 1584 & “ \\
\hline & btr10 & EU812796 & 1582 & $"$ \\
\hline & btr11 & EU812799 & 1584 & “ \\
\hline & btr16 & EU812792 & 1584 & " \\
\hline & btr17 & EU812801 & 1583 & $"$ \\
\hline & btr18 & EU812793 & 1584 & " \\
\hline \multirow[t]{4}{*}{ Unknown species } & btr19 & EU812802 & 1585 & " \\
\hline & btr22 & EU812797 & 1585 & " \\
\hline & btr23 & EU812800 & 1584 & " \\
\hline & ctr2 & EU812804 & 1585 & " \\
\hline
\end{tabular}


Table 1 List of specimens and size of ITS2 (bp) (Continued)

\begin{tabular}{|c|c|c|c|c|}
\hline & $\operatorname{ctr} 4$ & EU812803 & 1585 & $"$ \\
\hline & Т35.1 & EU812807 & 1584 & $"$ \\
\hline & T35.2 & EU812806 & 1585 & $"$ \\
\hline & bsk3 & EU812794 & 1585 & Sa Kaeo (Thailand) \\
\hline & S24.1 & EU812805 & 1585 & $"$ \\
\hline \multirow[t]{2}{*}{ Anopheles campestris } & csk10 & EU812808 & 1519 & Sa Kaeo (Thailand) \\
\hline & bsk34 & EU812809 & 1519 & $"$ \\
\hline
\end{tabular}

\section{Results}

\section{Sequence analysis and occurrence of repeat elements}

The fifty-one specimens identified as five members of the Barbirostris Group included 47 haplotypes, thus the majority of haplotypes contained a single specimen. The $\mathrm{G}+\mathrm{C}$ content varied between $52.3 \%$ and $55.9 \%$, similar to that found in other Anopheles species. All specimens showed an exceptionally large ITS2 amplicon. The size of ITS 2 in the members of the Barbirostris Group examined varied markedly in size: Clade I c. 1543 bp., Clade II 1727 bp, Clade-III had a similar ITS2 size 1730 bp but differed in 30 fixed substitutions. In Anopheles campestris ITS2 was 1519 bp long whilst in the unnamed member of the Barbirostris Group, Clade IV, ITS2 was 1583 bp. (Table 1).

The exceptional length of the ITS2 region was found to be due to the presence of repeated elements, located at the centre of the ITS2, occupying $55 \%$ to $61 \%$ of the total length of the spacer. The number and organization of the repeats varied between species (Figure 1 and Table 2). In Clade I, eight repeats, organized in two groups comprising four repeats each, were present. There was no length variation in these repeats, being 112 and 108 bp long for types 1 and 2, respectively. Repeats in Anopheles campestris were arranged in a similar manner, except that the last repeat at the 3 ' end turned into a non-repeated element. The presence of a single insertion/deletion event resulted in length variation in these repeats (Table 2). Anopheles vanderwulpi and Clade III showed a similar arrangement of nine repeated elements of three types. In both of these, sequences differed by several nucleotides. Non-repeated elements were also present in these species. Finally, the unknown species of the Barbirostris Group (Clade IV) contained five repeats of two types and varied sizes and three non-repeated elements (Figure 1). Most indels were located at the 3' end of the repeat sequences in species where indels were found.

Table 2 shows the nucleotide diversity of the different type of repeats found in Barbirostris Group members. The nucleotide diversity $\pi$ increased in direct relation to size variation and the presence of non-repeated elements. The lowest $\pi$ values were observed in repeats of Clade I and $A n$. campestris, whereas the unknown species Clade IV showed a high degree of polymorphism, particularly in type 2 repeats.

A GGGTGTG motif occurred at the $5^{\prime}$ end of most repeats. Although this motif was common to all species, in some cases variations of this motif were also observed

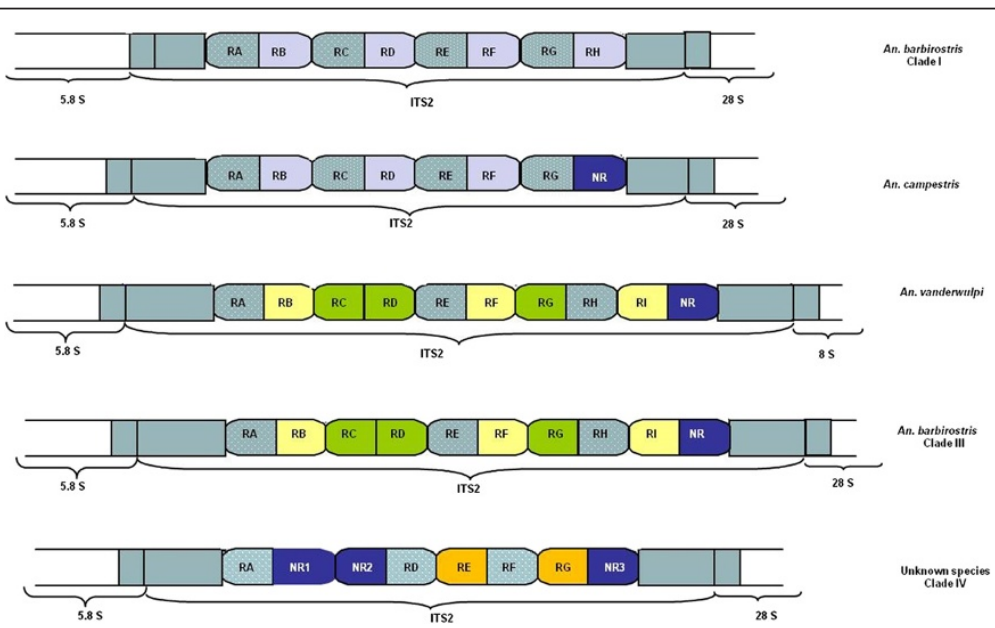

Figure 1 Showing location of repeats in ITS2 in five members of the Barbirostris group. Repeats (R) are symbolized with letters indicating position from $5^{\prime}$ end of ITS2 (A being closest). Non-repeated elements (NR) are located at 3' end. Similar filled patterns represent similar sequences. 
Table 2 Nucleotide diversity and sequence variation in repeats

\begin{tabular}{|c|c|c|c|c|c|}
\hline Repeats type & Repeats & $\mathrm{N}^{\circ}$ of SS & $\pi$ & $\mathrm{N}^{\circ}$ sites compared* & Sequence lengths \\
\hline Clade I type 1 & $A, C, E, G$ & 2 & 0.01 & 112 & 112 \\
\hline Clade I type 2 & $D, B, F, H$ & 5 & 0.02 & 108 & 108 \\
\hline An. campestris type 1 & $A, C, E, G$ & 4 & 0.02273 & 110 & $110(G), 111(A, C, E)$ \\
\hline An. campestris type 2 & $D, B, F$ & 9 & 0.05 & 112 & $112(F), 113(D, B)$ \\
\hline An. vanderwulpi type 1 & $A, E, H$ & 11 & 0.08 & 103 & $103(\mathrm{E}), 108(\mathrm{H}), 117(\mathrm{~A})$ \\
\hline An. vanderwulpi type 2 & $C, D, G$ & 6 & 0.04 & 102 & $102(\mathrm{D}), 105(\mathrm{G}), 107(\mathrm{C})$ \\
\hline An. vanderwulpi type 3 & $B, F, I$ & 6 & 0.04 & 98 & $99(\mathrm{~B}, \mathrm{~F}), 110(\mathrm{I})$ \\
\hline Clade III type 1 & $A, E, H$ & 17 & 0.11 & 107 & $107(\mathrm{E}), 113(\mathrm{H}), 116(\mathrm{~A})$ \\
\hline Clade III type 2 & $C, D, G$ & 7 & 0.05 & 101 & $103(\mathrm{D}, \mathrm{G}), 105(\mathrm{C})$ \\
\hline Clade III type 3 & $B, F, I$ & 7 & 0.05 & 98 & $99(B), 101(F), 113(I)$ \\
\hline Clade IV type 1 & $A, D, F$ & 5 & 0.04 & 93 & $97(F), 98(D), 116(A)$ \\
\hline Clade IV type 2 & $E, G$ & 16 & 0.14 & 114 & $114(E), 119(G)$ \\
\hline
\end{tabular}

SS: segregating sites.

*excluding sites with gaps/missing data.

(GGGTGGT in An. campestris, GGGTGGG in clade-I and An. vanderwulpi, GGGTGCG and TGGTGTG in the unknown member of the Barbirostris Subgroup). Using default parameters, most repeats formed long hairpin structures with a wide range of energy values. Results varied only in the number of hairpins that repeats formed, these being more evident in Clade I, Clade III and in An. campestris. At the base of the hairpin stem there was a motif GGGTGTG (or similar) at one side and a palindromic sequence on the other side of the stem. As an example Figure 2 shows the disposition of the hairpins in Clade I, at the lowest free energy value. Interestingly in this species each hairpin has a similar structure with three internal loops located close to the top of the hairpin (Figure 2). Stable RNA secondary structures are characterised by having low energy values. Energy values ranged from 593.6 to 629.4. Nevertheless, repeats formed at least 3 hairpin structures in all 25 resultant secondary structures, the only difference being the number of hairpins formed.

\section{Discussion}

Among members of the Barbirostris Group, An. barbirostris Clade III has the largest ITS2 recorded so far in Anopheles species ( 1730 bp). The large size of ITS2 in members of the Barbirostris Group (>1.5 kb) results from the presence of DNA insertions, comprising tandemrepeated elements of c.100 bp located in the centre of the spacer region. In the genus Anopheles the ITS2 is characterized by a very low level of polymorphism between species, even between cryptic species [24]. Despite reports of intraspecific and intra-individual variation [21], ITS2 is still widely used to infer phylogenetic relations among species $[30,31]$. In the Barbirostris Group, the presence of repeats did not affect tree topology, and this was consistent with results from COI analysis (10).

Given the high rate of substitution, we examined the possibility that the ITS2 of members of the Barbirostris Group were pseudogenes. This hypothesis was discarded for the following reasons: 1) There was a low rate of substitution in the flanking 5.8 and $28 \mathrm{~S}$ regions which were amplified, these comprise 90 nucleotides of the alignment; 2) Hairpin structures were formed for all species in high number, at the lowest free energy values. Pseudogenes in ribosomal spacers tend to accumulate random substitutions at high rates, whereas functional ITS regions show many hairpins with compact stable secondary structures [32]. Even when a point mutation was observed, it can be assumed that the structural integrity was not affected by the insertion of long repetitive elements, as large spacers seem to be less affected by mutations than shorter ones [33].

\section{Functional constraints on repeats}

Long ITS2 regions have been found in distant taxa including oomycetes [34] and amphipods [35]. They have also been reported in several arthropod species, including rhipicephaline ticks [23] and insects of various orders [36,37]. Repetitive DNA regions of considerable size have also been documented in the ITS2 of other Anopheles species $[8,19,20]$. Little has been written to explain the origin and function of repeated elements in ribosomal spacer regions. Secondary structure reconstructions show that repeats found in members of the Barbirostris Group form hairpin structures at a wide range of energy values. Since the Mfold server does not produce a single 'correct folding', many configurations obtained with the minimum free energy values were examined. It is known that hairpin structures are related to functional ITS 


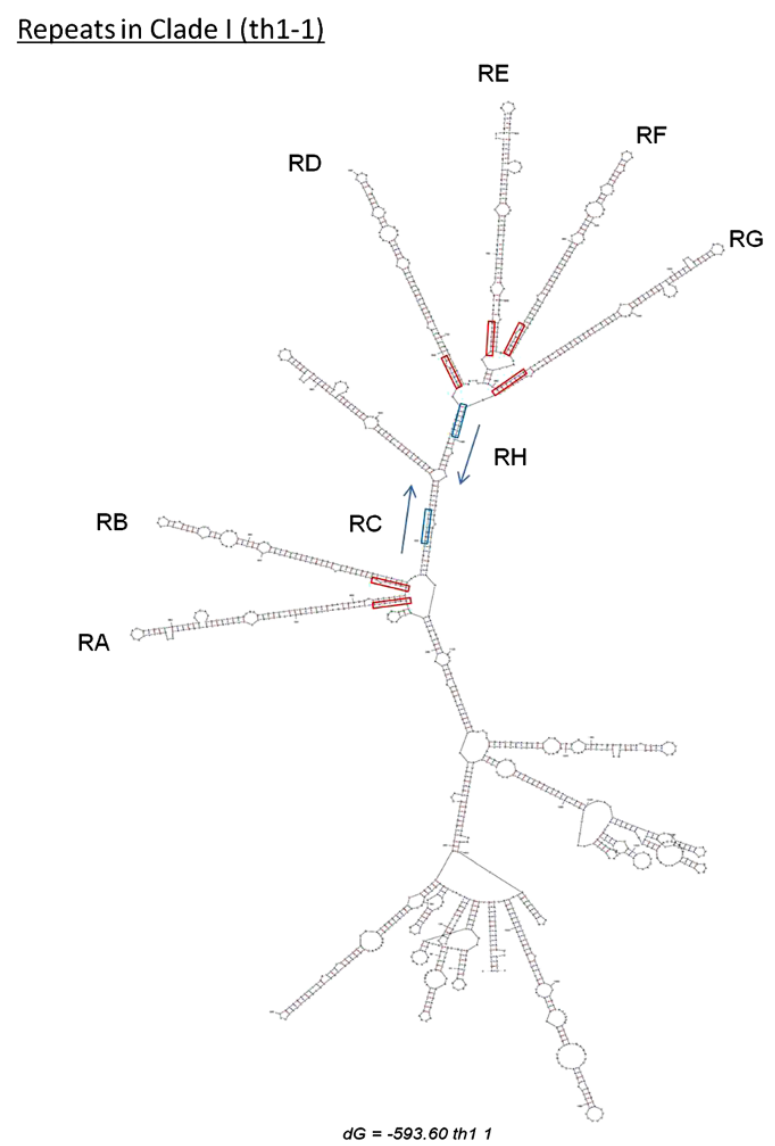

Figure 2 Secondary structure reconstruction of ITS2 in Clade-I of the An. barbirostris subgroup. The motif GGGTGTG is represented with a rectangle. Each one of the repeats $A, B, D, E, F$ and $\mathrm{G}$ form hairpin structures.

regions [32], although their functionality has not been fully elucidated. As stated by Paskewitz et al. [24] in their pioneering study of the secondary structure of ITS1 in the Anopheles gambiae complex, conservation of hairpin structures in a wide range of energy values may indicate the conservation of functional constraints, possibly related to the maturation of ribosomal subunits. Long stem-loop secondary structures are also formed by the repeats found in the ITS1 of members of the $A n$. punctulatus group [21]. However, the repeats found in the Barbirostris group showed no similarity to repeats in An. punctulatus or An. gambiae. Furthermore, a Blast search failed to reveal any similar sequence in GenBank. A possible explanation for this is that similarities to other insertional elements, e.g. transposable elements, may be obscured by the high rate of substitutions present in this spacer, as has been observed in other taxa [36].

Variation in other regions of the ribosomal gene has been reported as a result of adaptations to changes in the environment. Thus in the parthenogenetic Daphnia pulex, longer IGS regions provide these organisms with the plasticity required to adapt to different environments [38]. In Bryophytes, repetitive sequences in the rDNA appear to result from exposure to heavy metals. Whether insertion elements or adaptation to new environments affect the ITS2 region of members of the Barbirostris Group has yet to be determined.

ITS2 in members of the Barbirostris Group is subject to a high rate of evolutionary change. We observed a high rate of substitution, even at an intraspecific level and incomplete homogenization of repeats. Nevertheless, when comparing the rate of substitutions at an interspecies level, ITS2 seems to evolve through a pattern of concerted evolution. Accordingly, repeated elements found were imperfect copies of an original one. This has also been reported in the long spacers of other taxa $[22,39,40]$ and seems to be the result of replication slippage events [23].

Hairpin structures appear to facilitate subsequent processing [26]. Thus rRNA processing in yeast requires sequences and/or higher order structures within ITS2, an example of which would be the extensive folding of ITS2 sequences, bringing into juxtaposition those regions of mature $5.8 \mathrm{~S}$ and $25 \mathrm{~S}$ rRNA that must interact during processing. Thus rRNA processing requires sequences and/or higher order structures within ITS2. One example may be the extensive folding of the ITS2 sequences (see Figure 2), bringing into juxtaposition those regions in mature $5.8 \mathrm{~S}$ and $28 \mathrm{~S}$ rRNA that must interact but that are at a distance in the primary sequence.

Nucleotide diversity varied considerably among repeat types. It was higher in repeats from An.vanderwulpi, Clade III and Clade IV, where non-repeated elements were observed and the length of the repeats varied considerably. On the other hand, the degree of polymorphism was much lower in An.campestris and particularly in Clade I, where indels were absent. Concerted evolution is a potent force in the formation of ITS2, but there are cases where concerted evolution is incomplete, as seen in Anopheles longirostris from Papua New Guinea [33]. It is conceivable that concerted evolution is more effective in Clade I, leading to the stabilization of the repeats. This species is to date the most widely distributed member of the Anopheles barbirostris complex, having been reported in Thailand, Borneo (Indonesia), Vietnam [10] and in the islands of Sulawesi and Java, in the Indonesian archipelago [41]. Indels did not affect hairpin structures, as these were located at the 3' end, outside of hairpin structures.

We postulate that repeat elements were present in the common ancestor of the species of the Barbirostris Group, before members of the Group underwent subsequent speciation. This conclusion is based on two principal facts; firstly repeats in all species were flanked by a common motif (GGGTGTG and variants) and more 
importantly, the Bayesian analysis shows that type I repeats present in An. campestris form a monophyletic clade with their homologous repeats in Clade I.

\section{Conclusions}

We conclude that repeat elements were present in the common ancestor of species of the Barbirostris Group, before members of the Group underwent subsequent speciation. This conclusion is based on two principal observations; firstly repeats in all species are flanked by a common motif (GGGTGTG and variants) and more importantly, the Bayesian analysis shows that type I repeats present in An. campestris form a monophyletic clade with their homologous repeats in Clade I. Repeats form hairpin structures that are linked to the functional constraints on the internal transcribed spacer, since they are known to facilitate the processing of mature rRNA.

\section{Competing interests}

The authors declare that they have no competing interests.

\section{Authors' contributions}

CPE carried out the molecular studies and prepared draft Figures and Tables. HT assisted in the analysis of the data and their presentation. CPE provided an initial draft of the text. HT and CPE contributed equally to the final article and both authors read and approved the final manuscript.

\section{Author details}

'Department of Vector Biology, Liverpool School of Tropical Medicine, Pembroke Place, L3 5QA Liverpool, United Kingdom. ${ }^{2}$ Current address: Laboratory of Zoology. University of the Balearic Islands, Ctra de Valldemossa Km 7.5, 07122 Palma de Mallorca, Spain.

Received: 29 January 2014 Accepted: 7 March 2014

Published: 19 March 2014

\section{References}

1. Cote CA, Greer CL, Peculis BA: Dynamic conformational model for the role of ITS2 in pre-rRNA processing in yeast. RNA 2002, 8(6):786-797.

2. Coleman AW: ITS2 is a double-edged tool for eukaryote evolutionary comparisons. Trends Genet 2003, 19(7):370-375.

3. Good L, Intine RVA, Nazar RN: Interdependence in the processing of ribosomal RNAs in Schizosaccharomyces pombe. J Mol Biol 1997 273(4):782-788.

4. Dover G: Molecular drive - a cohesive mode of species evolution. Nature 1982, 299(5879):111-117.

5. Young I, Coleman AW: The advantages of the ITS2 region of the nuclear rDNA cistron for analysis of phylogenetic relationships of insects: a drosophila example. Mol Phyl Evol 2004, 30(1):236-242

6. Cornel AJ, Porter $\mathrm{CH}$, Collins FH: Polymerase chain reaction species diagnostic assay for Anopheles quadrimaculatus cryptic species (Diptera: Culicidae) based on ribosomal DNA ITS2 sequences. J Med Ent 1996, 33(1):109-116.

7. Manonmani A, Townson H, Adeniran $T$, Jambulingam P, Sahu S, Vijayakumar T: rDNA-ITS2 polymerase chain reaction assay for the sibling species of Anopheles fluviatilis. Acta Trop 2001, 78(1):3-9.

8. Wilkerson RC, Reinert JF, Cong L: Ribosomal DNA ITS2 sequences differentiate six species in the Anopheles crucians complex (Diptera: Culicidae). J Med Ent 2004, 41(3):392-401.

9. Loaiza JR, Bermingham E, Sanjur OI, Scott ME, Bickersmith SA, Conn JE: Review of genetic diversity in malaria vectors (Culicidae: Anophelinae). $J$ Med Epid Inf Gen Evol Infect Dis 2012, 12(1):1-12.

10. Paredes-Esquivel C, Donnelly MJ, Harbach RE, Townson H: A molecular phylogeny of mosquitoes in the Anopheles barbirostris subgroup reveals cryptic species: implications for identification of disease vectors. Mol Phyl Evol 2009, 50(1):141-151.
11. Reid JA: Anophelines of Malaya and Borneo. Stud Instit Med Res Mal, 31

12. Harrison B, Scanlon J: The subgenus Anopheles in Thailand (Diptera: Culicidae). Am Ent Instit 1975, 12:1-307. 1, pp iv +

13. Apiwathnasorn C, Prommongkol S, Samung Y, Limrat D, Rojruthai B: Potential for Anopheles campestris (Diptera : Culicidae) to transmit malaria parasites in Pa Rai subdistrict (Aranyaprathet, Sa Kaeo Province), Thailand. J Med Ent 2002, 39(4):583-586.

14. Reid JA: The Anopheles barbirostris group (Diptera, Culicidae). Bull Ent Res 1962, 53:1-57

15. Townson H, Dyer N, McAlister E, Satoto TBT, Bangs MJ, Harbach RE: Systematics of Anopheles barbirostris van der Wulp and a sibling species of the barbirostris complex (Diptera: Culicidae) in eastern Java, Indonesia. Syst Ent 2013, 38(1):180-191.

16. Lien JC, Kawengian BA, Partono F, Lami B, Cross JH: Brief survey of mosquitos of South Sulawesi, Indonesia, with special reference to identity of Anopheles barbirostris (Diptera-Culicidae) from Margolembo area. J Med Ent 1977, 13(6):719-727.

17. Reid JA, Harrison BA, Atmosoedjono S: Variation and vector status in Anopheles barbirostris. Mosquito Systematics 1979, 11:235-250.

18. Fu Q, Matsumoto $Y$, Matsumura M, Hirai Y, Sato Y, Noda H: Presence of a short repeat sequence in internal transcribed spacer (ITS) 1 of the rRNA gene of Sogatella furcifera (Hemiptera: Delphacidae) from geographically different populations in Asia. Appl Ent Zool 2012, 47(2):95-101.

19. Kampen H: The ITS2 ribosomal DNA of Anopheles beklemishevi and further remarks on the phylogenetic relationships within the Anopheles maculipennis group of species (Diptera : Culicidae). Parasit Res 2005, 97(2):118-128

20. Brelsfoard CL, Fritz GN, Rodriguez R: Sequence analysis of the rDNA internal transcribed spacer 2 and polymerase chain reaction identification of Anopheles fluminensis (Diptera: Culicidae: Anopheles) in Bolivia. J Med Ent 2006, 43(3):460-466.

21. Bower JE, Cooper RD, Beebe NW: Internal repetition and intraindividual variation in the rDNA ITS1 of the Anopheles punctulatus group (Diptera: Culicidae): multiple units and rates of turnover. J Mol Evol 2009, 68(1):66-79.

22. Murrell A, Campbell NJH, Barker SC: Recurrent gains and losses of large (84-109 bp) repeats in the rDNA internal transcribed spacer 2 (ITS2) of rhipicephaline ticks. Insect Mol Biol 2001, 10(6):587-596.

23. Hlinka O, Murrell A, Barker SC: Evolution of the secondary structure of the rRNA internal transcribed spacer 2 (ITS2) in hard ticks (Ixodidae, Arthropoda). Heredity 2002, 88:275-279.

24. Paskewitz SM, Wesson DM, Collins FH: The internal transcribed spacers of ribosomal DNA in five members of the anopheles gambiae species complex. Insect Mol Biol 1993, 2(4):247-257.

25. Thompson JD, Higgins DG, Gibson TJ: CLUSTALW: Improving the sensitivity of progressive multiple sequence alignment through sequence weighting, position-specific gap penalties and weight matrix choice. Nucl Acids Res 1994, 22(22):4673-4680.

26. Rozas J, Librado P, Sanchez-Del B, Messeguer X, Rozas R: DnaSP version 5.10.1, 4 March 2010: an integrated program for molecular population genetics and molecular evolution analysis. [http://www.ub.edu/dnasp/]

27. Nei M, Li WH: Mathematical-model for studying genetic-variation in terms of restriction endonucleases. Proc Natl Acad Sci U S A 1979, 76(10):5269-5273.

28. Zuker M: Mfold web server for nucleic acid folding and hybridization prediction. Nucl Acids Res 2003, 31(13):3406-3415.

29. Ronquist F, Huelsenbeck JP: MrBayes 3: Bayesian phylogenetic inference under mixed models. Bioinformatics 2003, 19(12):1572-1574.

30. Paredes-Esquivel C, Harbach RE, Townson H: Molecular taxonomy of members of the Anopheles hyrcanus group from Thailand and Indonesia. Med Vet Ent 2011, 25(3):348-352.

31. Ndo C, Simard F, Kengne P, Awono-Ambene P, Morlais I, Sharakhov I, Fontenille D, Antonio-Nkondjio C: Cryptic genetic diversity within the Anopheles nili group of malaria vectors in the equatorial forest area of Cameroon (central Africa). PLoS One 2013, 8(3):e58862. doi:10.1371/journal.pone.0058862.

32. Buckler ES, Ippolito A, Holtsford TP: The evolution of ribosomal DNA: divergent paralogues and phylogenetic implications. Genetics 1997, 145(3):821-832.

33. Alquezar DE, Hemmerter S, Cooper RD, Beebe NW: Incomplete concerted evolution and reproductive isolation at the rDNA locus uncovers nine cryptic species within Anopheles longirostris from Papua New Guinea. BMC Evol Biol 2010, 10:392. doi:10.1186/1471-2148-10-392. 
34. Thines $\mathrm{M}$ : Characterisation and phylogeny of repeated elements giving rise to exceptional length of ITS2 in several downy mildew genera (Peronosporaceae). Fungal Genet Biol 2007, 44(3):199-207.

35. Kornobis E, Palsson S: The ITS region of groundwater amphipods: length secondary structure and phylogenetic information content in Crangonyctoids and Niphargids. J Zool Syst \& Evol Res 2013, 51(1):19-28.

36. von der Schulenburg JHG, Hancock JM, Pagnamenta A, Sloggett JJ, Majerus MEN, Hurst GDD: Extreme length and length variation in the first ribosomal internal transcribed spacer of ladybird beetles (Coleoptera: Coccinellidae). Mol Biol Evol 2001, 18(4):648-660.

37. Oh HK, Yoon HJ, Kim MJ, Jeong HU, Kim SR, Hwang JS, Bae CH, Kim I: ITS2 Ribosomal DNA sequence variation of the bumblebee, Bombus ardens (Hymenoptera: Apidae). Genes \& Genomics 2009, 31(4):293-303.

38. Gorokhova E, Dowling TE, Weider LJ, Crease TJ, Elser JJ: Functional and ecological significance of rDNA intergenic spacer variation in a clonal organism under divergent selection for production rate. Proc of Roy SoC London Ser B-Biol Sci 2002, 269(1507):2373-2379.

39. Thines M, Komjati H, Spring O: Exceptional length of ITS in Plasmopara halstedii is due to multiple repetitions in the ITS2 region. Eur J Plant Pathol 2005, 112(4):395-398.

40. Choi YJ, Hong SB, Shin HD: Extreme size and sequence variation in the ITS rDNA of Bremia lactucae. Mycopathologia 2007, 163(2):91-95.

41. Satoto TBT: Cryptic species within Anopheles barbirostris Van der Wulp, 1884 inferred from nuclear and mitochondrial gene sequence variation (Ph.D thesis). University of Liverpool; 2001

doi:10.1186/1756-3305-7-106

Cite this article as: Paredes-Esquivel and Townson: Functional

constraints and evolutionary dynamics of the repeats in the rDNA

internal transcribed spacer 2 of members of the Anopheles barbirostris

group. Parasites \& Vectors 2014 7:106.

\section{Submit your next manuscript to BioMed Central and take full advantage of:}

- Convenient online submission

- Thorough peer review

- No space constraints or color figure charges

- Immediate publication on acceptance

- Inclusion in PubMed, CAS, Scopus and Google Scholar

- Research which is freely available for redistribution 